\title{
Nuclear Power Plant Construction Scheduling Problem with Time Restrictions: A Particle Swarm Optimization Approach
}

\author{
Shang-Kuan Chen, ${ }^{1}$ Yen-Wu Ti, ${ }^{2}$ and Kuo-Yu Tsai ${ }^{3}$ \\ ${ }^{1}$ Department of Computer Science and Information Engineering, Yuanpei University of Medical Technology, Hsinchu 23568, Taiwan \\ ${ }^{2}$ Department of Computer Science and Information Engineering, Hwa Hsia University of Technology, No. 111, \\ Gong Jhuan Road, Chung Ho, Taipei 23568, Taiwan \\ ${ }^{3}$ Department of Applied Mathematics, Chinese Culture University, No. 55, Hwa-Kang Road, Yang-Ming-Shan, Taipei 11114, Taiwan
}

Correspondence should be addressed to Yen-Wu Ti; d91010@csie.ntu.edu.tw

Received 16 November 2015; Revised 25 January 2016; Accepted 2 February 2016

Academic Editor: Arkady Serikov

Copyright (c) 2016 Shang-Kuan Chen et al. This is an open access article distributed under the Creative Commons Attribution License, which permits unrestricted use, distribution, and reproduction in any medium, provided the original work is properly cited.

In nuclear power plant construction scheduling, a project is generally defined by its dependent preparation time, the time required for construction, and its reactor installation time. The issues of multiple construction teams and multiple reactor installation teams are considered. In this paper, a hierarchical particle swarm optimization algorithm is proposed to solve the nuclear power plant construction scheduling problem and minimize the occurrence of projects failing to achieve deliverables within applicable due times and deadlines.

\section{Introduction}

Many countries with existing nuclear power projects (Argentina, Armenia, Brazil, Bulgaria, China, Czech Rep., India, Pakistan, Romania, Russia, Slovakia, South Korea, South Africa, UAE, Ukraine, UK, and USA) have plans to build new reactors beyond those already under construction. In total, over 160 power reactors with a total net capacity of about 186,000 MWe are planned and over 300 more have been proposed [1]. Efficient scheduling of projects is a key component of effective utilization of construction companies and reactor builders. However, design-build contracts usually place a restrictive due time and deadline for their projects. That is, if the project is completed later than the due time but still meets the deadline, the owners may impose a penalty on the construction company and/or reactor builder, and if the project does not meet the deadline, the penalty is even greater. Further, this may damage the company's reputation and lose them future contracts. Hence, an efficient scheduling algorithm for nuclear power plant construction is highly beneficial. Nuclear power plant construction scheduling problems have been the subject of extensive research for several years in the optimization field, with the aim of practical application $[2,3]$.

In particular, the resource-constrained scheduling problem has been extensively studied as a key component of project scheduling analysis. Reliable construction schedules are vital for effective coordination across supply chains and various trades at the construction work face [4]. The main objective of solving this problem is to develop optimal schedules with regard to resource allocation (subject to resource availability), resource leveling, and project duration minimization or constriction within given deadlines [5]. Since the late 1950s, the critical path method (CPM) and program evaluation and review technique (PERT) have been intensively used by practitioners to plan and control largescale projects in the construction industry. In traditional CPM and PERT analysis, a major objective is to establish the feasible duration required to perform a specific project under the assumption of unlimited resources. However, in reality, resources, including crew size, equipment, and materials, are limited [6]. With an unreliable schedule, project participants 
are likely to neglect the official plan and work towards their own priorities, potentially leading to conflicts, low productivity, and considerable waste. Nuclear plant construction contractors often encounter projects that contain several identical or similar units, such as floors in multistory buildings, houses in housing developments, meters in pipelines, or stations in a nuclear plant. These multiunit projects are characterized by repeated activities, which usually arise from the subdivision of a generalized activity into specific activities associated with particular units [7]. Activities that repeat from unit to unit require a construction schedule that facilitates the uninterrupted flow of resources (i.e., worker teams) from one unit to the next, because it is often this requirement that establishes activity starting times and determines the overall project duration. Hence, uninterrupted resource utilization is an extremely important issue [7].

The generalized model of nuclear power plant construction scheduling is valuable in that it can be incorporated into commercial project management software, allowing project managers to use advanced computational methods for practical applications [8]. The scheduling problem posed by multiunit projects with repeated activities is equivalent to the minimization of the project duration subject to resource continuity constraints and technical precedence constraints. The uninterrupted deployment of resources is not addressed by CPM, nor is it addressed by its resource-oriented extensions, such as time-cost trade-off, limited resource allocation, and resource leveling. However, the requirement for uninterrupted utilization of resources from an activity in one unit to the same (repeating) activity in the next unit is explicitly recognized by several scheduling methodologies that have been available for many years $[7,9,10]$.

A variety of methods and algorithms have been produced via research for the resource-constrained scheduling problem. These can be classified as exact methods (linear/integer or dynamic programming), heuristic algorithms, and metaheuristic or evolutionary algorithms. Exact methods develop mathematical relationships for the objective function and the problem constraints and solve the problem using a linear or dynamic optimization technique [5, 11-15].

Traditional construction project planning and scheduling has been widely criticized. Leu and Yang (1999) applied a genetic algorithms approach for construction scheduling based on multicriteria computational optimal scheduling model. They provide the optimal combination of construction durations, resource amounts, minimum direct project costs, and minimum project duration under the constraint of limited resources [16]. Senouci and Eldin (2004) present an augmented Lagrangian genetic algorithm model for resource scheduling. They considered all precedence relationships, multiple crew strategies, total project cost minimization, and time-cost trade-off [17]. Agarwal et al. (2011) proposed a neurogenetic approach which is a hybrid of genetic algorithms and neural-network approaches for solving the resourceconstrained project scheduling problem. They found that the hybrid approach gives better solutions than either approach independently for the same number of shared iterations [18]. A common criticism is that the theory and practice focus on contract management and cost control rather than production at the construction work face. However, increasingly many production companies need to change their production lines to meet the requirements of customers. Production scheduling and vehicle routing are integrated to solve a production issue with a timing requirement. In the integrated production scheduling and vehicle routing problem, each order is generally defined by its dependent setup time and processing time for the production process and by its delivery time and time window for the delivery process.

The building of a nuclear power plant requires the cooperation of construction companies and reactor builders. Thus, to obtain a complete solution of the construction scheduling problem, this paper integrates the problems of integrated production scheduling and vehicle routing with the nuclear power plant construction scheduling problem. Recently, particle swarm optimization algorithm and variable neighborhood search have performance well across a wide range of job scheduling problems [19-21]. We develop a hierarchical variable neighborhood search algorithm for solving nuclear power plant construction scheduling problem. Variable neighborhood search (VNS) is a metaheuristic, or a framework for building heuristics, based upon systematic changes of neighborhoods both in descent phase, to find a local minimum, and in perturbation phase to emerge from the corresponding valley. It travels neighborhoods of the current solution node and travels to a new node if and only if it is a better solution. Therefore, the total waiting time can be efficiently shortened for nuclear power plant construction. Throughout, we consider key issues, including identical construction teams, buildings at nuclear power plant sites, sequence-dependent preparing times, identical teams to install reactors, and time windows to wait for construction. Owing to the extreme complexity of this combined problem, we only test small-sized examples to confirm our algorithm's ability to obtain an optimal solution. We use the commercial software LINGO 11.0, which is based on the branch \& bound method, to find the optimal solution.

The remainder of this paper is organized as follows. Section 2 describes the proposed mathematical model. The proposed method is shown in Section 3. Experimental results are provided in Section 4. Finally, brief conclusions are given in Section 5.

\section{The Proposed Mathematical Model}

We adopt an integer linear programming formulation for the mathematical model of the nuclear power plant construction scheduling problem. The parameters are defined as follows:

$p t_{i}=$ the construction time of buildings at the nuclear power plant $i$.

$s t_{0 i}=$ the preparation time of the first nuclear power plant $i$.

$s t_{i j}=$ the preparation time of buildings at the nuclear power plant $j$ constructed after nuclear power plant $i$.

$t r_{0 i}=$ the installation time for the reactors of nuclear power plant $i$. 
$t r_{i j}=$ the installation time for the reactors of nuclear power plant $j$, after nuclear power plant $i$ installed reactors.

$a_{i}=$ the lower bound of the time window of nuclear power plant $i$.

$b_{i}=$ the upper bound of the time window of nuclear power plant $i$.

$w_{i}=$ the weight of nuclear power plant $i$.

$u_{i}=$ the number of reactors of nuclear power plant $i$.

$\operatorname{cap}_{k}=$ the capacity of reactor installation team $k$.

$$
\begin{aligned}
& M=\text { a large number. } \\
& n=\text { the number of nuclear power projects. }
\end{aligned}
$$

Note that $a$ and $b$ are arbitrary variables. If we wish to ensure that value $a$ is much larger than value $b$, we restrict $a-M b>0$ in our program.

Two sets of binary variables, $z a$ and $x a$, are defined to handle the construction of buildings at the nuclear power plants, and two more sets of binary variables, $z b$ and $x b$, are defined to handle the installation of reactors, as follows:

$z a_{i}^{m}= \begin{cases}1 & \text { if nuclear power plant } i \text { is constructed by construction worker team } m \\ 0 & \text { otherwise, }\end{cases}$

$x a_{i j}^{m}= \begin{cases}1 & \text { if construction worker team } m \text { builds nuclear power plant } i \text {, then builds nuclear power plant } j \\ 0 & \text { otherwise, }\end{cases}$

$z b_{i}^{k}= \begin{cases}1 & \text { if the reactor of nuclear power plant } i \text { is installed by reactor installation team } k \\ 0 & \text { otherwise, }\end{cases}$

$x b_{i j}^{k}$

$= \begin{cases}1 & \text { if reactor installation team } k \text { installs the reactor of nuclear power plant } i \text {, then installs the reactor of nuclear power plant } j \\ 0 & \text { otherwise. }\end{cases}$

The continuous variables, $T 1_{i}, T 2_{i}, T 3_{i}^{k}$, and $T_{i}$, are defined to handle the time of finishing construction of the building of nuclear power plant $i$, the start time of installation of reactor of nuclear power plant $i$, the finish time of reactor installation team $k$ to install the reactor of nuclear power plant $i$, and the delay of nuclear power plant project $i$, respectively.

The objective function is: $\min \sum_{i=1}^{n} w_{i} T_{i}$, such that

$$
\begin{aligned}
& \sum_{m} z a_{i}^{m}=1 \quad \forall i, \\
& x a_{i j}^{m} \leq z a_{i}^{m} \quad i \neq j, \forall i, j, m, \\
& x a_{i j}^{m} \leq z a_{j}^{m} \quad i \neq j, \forall i, j, m, \\
& T 1_{i} \geq s t_{o i} z a_{i}^{m}+p t_{i} z a_{i}^{m} \quad \forall i, m, \\
& T 1_{j}-T 1_{i}+M\left(3-x a_{i j}^{m}-z a_{i}^{m}-z a_{j}^{m}\right) \geq s t_{i j}+p t_{j} \\
& T 1_{i}-T 1_{j}+M\left(2+x a_{i j}^{m}-z a_{i}^{m}-z a_{j}^{m}\right) \geq s t_{j i}+p t_{i} \\
& T 1_{i} \geq 0 \quad \forall i, \quad i \neq j, \quad \forall i, j, m, \\
& s t_{o i} \geq 0 \quad \forall i,
\end{aligned}
$$

$$
\begin{aligned}
& s t_{i j} \geq 0 \quad i \neq j, \forall i, j, \\
& p t_{i} \geq 0 \quad \forall i, \\
& \sum_{k} z b_{i}^{k}=1 \quad \forall i, \\
& x b_{i j}^{k} \leq z b_{i}^{k} \quad i \neq j, \forall i, j, k, \\
& x b_{i j}^{k} \leq z b_{j}^{k} \quad i \neq j, \forall i, j, k, \\
& T 3_{i}^{k} \leq M \times z b_{i}^{k} \quad \forall i, k, \\
& T 3_{i}^{k}-T 1_{j}+M\left(2-z b_{i}^{k}-z b_{j}^{k}\right) \geq 0 \quad \forall i, j, k, \\
& T 3_{i}^{k}-T 3_{j}^{k} \leq M\left(2-z b_{i}^{k}-z b_{j}^{k}\right) \quad \forall i, j, k, \\
& T 2_{i} \geq T 3_{i}^{k}+t r_{o i} z b_{i}^{k} \quad \forall i, k,
\end{aligned}
$$

$$
i \neq j, \forall i, j, k
$$$$
T 2_{i}-T 2_{j}+M\left(2+x b_{i j}^{k}-z b_{i}^{k}-z b_{j}^{k}\right) \geq t r_{j i}
$$$$
i \neq j, \forall i, j, k \text {, }
$$

$\sum_{i} u_{i} z b_{i}^{k} \leq \operatorname{cap}_{k} \quad \forall k$ 


$$
\begin{aligned}
& T 2_{i} \geq a_{i} \quad \forall i \\
& T_{i} \geq T 2_{i}-b_{i} \quad \forall i, \\
& T_{i} \geq 0 \quad \forall i \\
& T 2_{i} \geq 0 \quad \forall i, \\
& t r_{o i} \geq 0 \quad \forall i, \\
& t r_{i j} \geq 0 \quad i \neq j, \quad \forall i, j, \\
& T 3_{i}^{k} \geq 0 \quad \forall i, k .
\end{aligned}
$$

Constraint (2) ensures that each nuclear power plant is constructed by only one construction team. Constraints (3) and (4) ensure that projects are scheduled after they are assigned to a construction team. Constraint (5) ensures that the finish time of a project is greater than preparation time plus construction time. Constraints (6) and (7) ensure the minimization of the project finish time. Constraints (8), (9), (10), and (11) ensure that operation time is greater than zero. Constraint (12) ensures that each reactor is installed by only one reactor installation team. Constraints (13) and (14) ensure that reactor installations are scheduled after they are assigned to a reactor installation team. Constraint (15) ensures that a reactor installation team only starts work after a reactor has been assigned to them. Constraints (16) and (17) ensure that reactor installation teams have enough time to complete the job. Constraints (18), (19), and (20) calculate the start time of the installation of the reactor of the nuclear power plant. Constraints (23) and (24) present the restrictions of delay time.

\section{The Proposed Method}

In this section, particle swarm optimization and a variable neighborhood search method are adapted to solve the nuclear power plant construction scheduling problem. The mathematical model described in the previous section governs the variables of this problem. We designed hierarchical variable neighborhood search (VNS) methods for both building construction and reactor installation. The adapted VNS method includes the following at both the construction team level and the reactor installation team level: project exchange on a construction worker team, construction preparation, project insertion on a construction worker team, and construction worker team exchange. For initial solutions, we randomly generate $p$ particles by a set of random numbers. Each particle then searches for better solutions using VNS. If no better solution can be found by VNS, the particles change their directions according to their personal best solution and the global best solution. The searching process then continues until a terminal condition is reached. The algorithm is summarized as follows.

Step 1. Generate $k$ construction team particles with a set of locations $x m_{1}, x m_{2}, \ldots, x m_{k}$, and a set of velocities $v m_{1}, v m_{2}, \ldots, v m_{k}$. Then, $x m_{i}=\left\{x m_{i 1}, x m_{i 2}\right\}$ and $v m_{i}=$ $\left\{v m_{i 1}, v m_{i 2}\right\}$, where $x m_{i 1}$ refers to the construction sequence and $x m_{i 2}$ refers to the construction team assigned to this construction sequence.

Step 2. Set the following parameters: the inertia weight $w$, the weight of the personal best solution $c_{1}$, the weight of the global best solution $c_{2}$, and random parameters $r_{1}$ and $r_{2}$ generated by a uniform distribution $U(0,1)$.

Step 3. Update the personal best solution $p b m_{i j}$ and the global best solution $\mathrm{gbm}_{j}$.

Step 4. Generate $k$ reactor installation team particles with a set of locations $x v_{1}, x v_{2}, \ldots, x v_{k}$, and with a set of velocities $v v_{1}, v v_{2}, \ldots, v v_{k}$. Then, $x v_{i}=\left\{x v_{i 1}, x v_{i 2}\right\}$ and $v v_{i}=\left\{v v_{i 1}, v v_{i 2}\right\}$, where $x v_{i 1}$ refers to the routing sequence and $v v_{i 2}$ refers to the reactor installation team assigned to this routing sequence.

Step 5. For each particle, apply VNS to find the personal best solution $p b v_{i j}$.

Step 6. From the set of personal best solutions, determine the global best solution $g b v_{j}$.

Step 7. Update the velocities and locations of the reactor installation scheduling according to the following:

$$
\begin{aligned}
v v_{i j}= & w v v_{i j}+c_{1} r_{1}\left(p b v_{i j}-x v_{i j}\right) \\
& +c_{2} r_{2}\left(g b v_{j}-x v_{i j}\right), \\
x v_{i j}= & x v_{i j}+v v_{i j} .
\end{aligned}
$$

Step 8. If these do not meet the local terminal condition, return to Step 5.

Step 9. Update the velocities and locations regarding building construction scheduling according to the following:

$$
\begin{aligned}
v m_{i j}= & w v m_{i j}+c_{1} r_{1}\left(p b m_{i j}-x m_{i j}\right) \\
& +c_{2} r_{2}\left(g b m_{j}-x m_{i j}\right), \\
x m_{i j}= & x m_{i j}+v m_{i j} .
\end{aligned}
$$

Step 10. If these meet the terminal condition, stop; otherwise, return to Step 3.

Various parameter settings such as velocity and inertia can be justified as follows from the equations

$$
\begin{aligned}
v v_{i j}= & w v v_{i j}+c_{1} r_{1}\left(p b v_{i j}-x v_{i j}\right) \\
& +c_{2} r_{2}\left(g b v_{j}-x v_{i j}\right), \\
v m_{i j}= & w v m_{i j}+c_{1} r_{1}\left(p b m_{i j}-x m_{i j}\right) \\
& +c_{2} r_{2}\left(g b m_{j}-x m_{i j}\right) .
\end{aligned}
$$

Consider the following:

(1) The weight of inertia $w$ : this is a parameter that gives a weight to the velocity in previous iteration. 
TABLE 1: Bounds to generate the experimental parameters.

Parameter (Lower bound, upper bound)

Construction time of the building of nuclear power plant

Weight of order

Preparation time (first)

Preparation time (dependent)

Number of reactors

Capacity of reactor installation team

Lower bound of time window

Upper bound of time window

Reactor installation time (first)

Reactor installation time (dependent)

TABLE 2

\section{Construction time of the building of nuclear power plant}

46

Preparation time (first)

10

Lower bound of time window

73

Upper bound of time window

142

Preparation time (dependent)

0

9

15

17

11

Reactor installation time

34

Reactor installation time (dependent)

0

43

29

24

36

Weight of order

2
50

17

85

144

11

0

11

8

9

41

29

0

50

29

44

2
39

14

78

138

163

17

$9-12$

0

8

8

29

41

47

0

48

25

1
169

35

15

18

92

69

12

9

13

18

0

18

36

36

30

41

0 2
(2) The random numbers $r_{1}$ and $r_{2}$ : uniform distribution on $[0,1)$.

(3) The weight of personal best solution $c_{1}$ : this is a parameter that gives a weight to the personal best solution.

(4) The weight of global best solution $c_{2}$ : this is a parameter that gives a weight to the global best solution.

\section{Experimental Results}

We used the optimization software LINGO 11.0 to obtain the optimum solution. We generate the parameters as random numbers with upper and lower bounds, as listed in Table 1.
This experiment has a set of parameters for eight orders. The parameters are generated as in Table 2. If the number of construction teams of the building of nuclear power plant equals 2 and that of reactor installation team equals 2 , then the best solution of the proposed nuclear power plant construction scheduling problem based on the above parameters is shown as follows.

The best schedule (construction of the building of nuclear power plant) is as follows:

Construction team 1 (plant $2==>$ plant 3$)$.

Construction team 2 (plant $1==>$ plant $5==>$ plant $4)$. 
TABLE 3: The experimental results (minimal tardiness time).

\begin{tabular}{|c|c|c|c|c|c|c|}
\hline \multirow[t]{2}{*}{ Minimal tardiness time } & \multicolumn{6}{|c|}{$\begin{array}{c}\text { (Number of nuclear power plants, number of construction } \\
\text { teams, and number of reactor installation teams) }\end{array}$} \\
\hline & $(6,1,1)$ & $(6,2,1)$ & $(6,1,2)$ & $(6,2,2)$ & $(6,2,3)$ & $(6,3,2)$ \\
\hline The optimal solution of LINGO 11 & 2004 & 740 & 1008 & 265 & 151 & 72 \\
\hline The solution of the proposed method & 2004 & 740 & 1008 & 265 & 151 & 72 \\
\hline
\end{tabular}

TABLE 4: The experimental results (time spanning).

\begin{tabular}{|c|c|c|c|c|c|c|}
\hline \multirow[t]{2}{*}{ Time spanning (seconds) } & \multicolumn{6}{|c|}{$\begin{array}{c}\text { (Number of nuclear power plants, number of construction } \\
\text { teams, and number of reactor installation teams) }\end{array}$} \\
\hline & $(6,1,1)$ & $(6,2,1)$ & $(6,1,2)$ & $(6,2,2)$ & $(6,2,3)$ & $(6,3,2)$ \\
\hline The optimal solution of LINGO 11 & 534 & 200 & 196 & 413 & 386 & 568 \\
\hline The solution of the proposed method & 1.56 & 0.82 & 1.04 & 1.32 & 0.89 & 1.65 \\
\hline
\end{tabular}

TABLE 5: The range of generating instance.

\begin{tabular}{lc}
\hline Parameter & $\begin{array}{c}\text { (Lower bound, upper } \\
\text { bound) }\end{array}$ \\
\hline $\begin{array}{l}\text { Construction time of the building of } \\
\text { nuclear power plant }\end{array}$ & $(25,50)$ \\
Weight of order & $(1,2)$ \\
Preparation time (first) & $(10,20)$ \\
Preparation time (dependent) & $(8,18)$ \\
Number of reactors & $(1,3)$ \\
Capacity of reactor installation team & $(16,21)$ \\
Lower bound of time window & $(65,99)$ \\
Upper bound of time window & $(95,180)$ \\
Reactor installation time (first) & $(18,45)$ \\
Reactor installation time (dependent) & $(20,50)$ \\
\hline
\end{tabular}

The best schedule (reactor installation) is as follows:

Reactor installation team 1 (plant $5==>$ plant $4==>$ plant 3).

Reactor installation team 2 (plant $2==>$ plant 1 ).

The minimum delay: 162 .

The minimum delay obtained by the proposed method is the same as that obtained by LINGO 11.0. We summarize the results of repeating experiments six times in Tables 3, 4, and 5.

The instance is used for above experiments (see Table 6). From the results, we can see that the increase of construction teams is more effective than the increase of reactor installation team. This is because the plant construction time is usually much smaller than the reactor installation time. Furthermore, we can see that when the range between lower bound and upper bound becomes wider, the performance becomes better. As the range between lower bound and upper bound of a plant becomes wider, more construction teams and reactor installation teams are able to build it, which gives our method more room to schedule the teams properly to achieve the minimum delay. The complexity of the proposed algorithm is $O\left(n^{2}\right)$, where $n$ is the number of nuclear power plants.
The CPU time of the proposed method depends on the number of nuclear power plants and the number of reactor builders. For the example shown in Table 4, the CPU time of the proposed method is much smaller than that of practicing in Lingo 11.

Besides the randomly generated instances, we use a realworld instance in order to assess that our proposed method would perform well in practical conditions. From 2009 to present, China General Nuclear (CGN) Power Group signed 14 agreements with Hongyanhe, Ningde, Yangjiang, Taishan, Fangchenggang, and Xianning to develop nuclear projects totaling 23 reactors, and the models of reactor are listed in Table 7 [1].

We generate the parameters according to the sequential of agreements, the number of reactors, and the reactor models, as listed in Tables 8 and 9. We set the lower bound and upper bound of time window by estimated time that the reactors are expected to be installed. In order to simplify the analysis we will regard the Hongyanhe unit 4 as N1, Ningde unit 4 as N2, and so on, and our discussion will not be affected.

If the number of construction teams of the building of nuclear power plant equals 5 and that of reactor installation team equals 5 , then the best solution of the proposed nuclear power plant construction scheduling problem based on the plans of CG is shown as follows.

The best schedule (construction of the building of nuclear power plant) is as follows:

$$
\begin{aligned}
& \text { Construction team } 1(\mathrm{~N} 10==>\mathrm{N} 14==>\mathrm{N} 12) . \\
& \text { Construction team } 2(\mathrm{~N} 7==>\mathrm{N} 2==>\mathrm{N} 4) . \\
& \text { Construction team } 3(\mathrm{~N} 3==>\mathrm{N} 9==>\mathrm{N} 13) \\
& \text { Construction team } 4(\mathrm{N1}==>\mathrm{N} 8==>\mathrm{N} 11) \\
& \text { Construction team } 5(\mathrm{~N} 5==>\mathrm{N} 6) .
\end{aligned}
$$

The best schedule (reactor installation) is as follows:

Reactor installation team $1(\mathrm{~N} 2==>\mathrm{N} 1==>\mathrm{N} 5==>$ N6).

Reactor installation team $2(\mathrm{~N} 14==>\mathrm{N} 13==>$ N11).

Reactor installation team $3(\mathrm{~N} 4==>\mathrm{N} 12)$. 
TABLE 6

Construction time of the building of nuclear power plant

49

Preparation time (first)

14

Lower bound of time window

82

Upper bound of time window

176

Preparation time (dependent)

0

9

9

14

9

13

Reactor installation time

23

Reactor installation time (dependent)

0

37

49

40

26

40

Weight of order

1
44

15

88

115

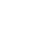

15

0

10

10

14

14

36

19

43

0

46

34

48

26

1
44

12

99

111

18

17

11

13

16

10

35

49

0

28

43

44
43

20

74

124

13

8

0

0

14

17

24

24

28

24

0

43

21
43

17

67

155

1
TABLE 8: The experimental parameters (lower bounds, upper bounds, and weights).

\begin{tabular}{lc}
\hline Plant & Reactor model \\
\hline Hongyanhe unit 4 & CPR-1000 \\
Ningde unit 4 & CPR-1000 \\
Yangjiang unit 4 & CPR-1000+ \\
Taishan units $1 \& 2$ & EPR \\
Fangchenggang unit 2 & CPR-1000 \\
Yangjiang units 5 \& 6 & ACPR-1000 \\
Hongyanhe units $5 \& 6$ & ACPR-1000 \\
Fangchenggang units $3 \& 4$ & Hualong 1 \\
Ningde units $5 \& 6$ & Hualong 1 \\
Lufeng units $1 \& 2$ & AP-1000 \\
Fangchenggang units $5 \& 6$ & Hualong 1 \\
Huizhou units $1 \& 2$ & AP-1000 \\
Taishan units units $3 \& 4$ & EPR \\
Xianning units $1 \& 2$ & AP-1000 \\
\hline
\end{tabular}

Reactor installation team 4 (N9 ==> N10).

Reactor installation team $6(\mathrm{~N} 3==>\mathrm{N} 8==>\mathrm{N} 7)$.

The minimum delay: 111.1.

On the other hand, Generic Algorithm (GA) is one of heuristic algorithms for solving NP-hard problem. Using the similar encoding of particle in PSO, we use a GA for solving

\begin{tabular}{lc}
\hline Plant & $\begin{array}{c}\text { (Lower bound, upper bound, weight, } \\
\text { construction time, and reactor installation time) }\end{array}$ \\
\hline N1 & $(40,86,1,40,1)$ \\
N2 & $(40,84,1,40,1)$ \\
N3 & $(40,72,1,40,1)$ \\
N4 & $(46,96,0.5,40,1.5)$ \\
N5 & $(40,72,1,46,1.5)$ \\
N6 & $(40,84,0.5,42,1.5)$ \\
N6 & $(40,72,0.5,42,1.5)$ \\
N7 & $(48,72,0.5,42,1.5)$ \\
N8 & $(48,72,0.5,42,1.5)$ \\
N9 & $(48,72,0.5,42,1.5)$ \\
N10 & $(48,72,0.5,46,1.5)$ \\
N11 & $(48,120,0.5,42,1.5)$ \\
N12 & $(48,120,0.2,46,1.5)$ \\
N13 & $(48,120,0.2,42,1.5)$ \\
N14 & $(48,120,0.2,42,1.5)$ \\
\hline
\end{tabular}

the Nuclear power plant construction scheduling problem. The selection, crossover, and mutation operators are designed for the GA. Using the same number of iterations, 1000 times, 
TABle 9: Dependent time.

\begin{tabular}{lcccccccccccccc}
\hline & N1 & N2 & N3 & N4 & N5 & N6 & N7 & N8 & N9 & N10 & N11 & N12 & N13 & N14 \\
\hline N1 & & 1 & 2 & 3 & 1 & 3 & 3 & 2 & 2 & 3 & 2 & 3 & 3 & 3 \\
N2 & 1 & & 2 & 3 & 1 & 3 & 3 & 2 & 2 & 3 & 2 & 3 & 3 & 3 \\
N3 & 2 & 2 & & 3 & 2 & 3 & 3 & 2 & 2 & 3 & 2 & 3 & 3 & 3 \\
N4 & 3 & 3 & 3 & & 1 & 2 & 2 & 2 & 3 & 1 & 3 & 3 & 1 & 2 \\
N5 & 1 & 1 & 2 & 1 & & 2 & 2 & 2 & 2 & 3 & 1 & 3 & 3 & 2 \\
N6 & 3 & 3 & 3 & 2 & 2 & & 1 & 2 & 3 & 2 & 3 & 3 & 2 & 2 \\
N7 & 3 & 3 & 3 & 2 & 2 & 1 & & 1 & 2 & 2 & 3 & 1 & 2 \\
N8 & 2 & 2 & 2 & 2 & 2 & 2 & 1 & & 1 & 3 & 1 & 2 & 2 \\
N9 & 2 & 2 & 2 & 3 & 2 & 3 & 2 & 1 & & 2 & 1 & 2 & 2 \\
N10 & 3 & 3 & 3 & 1 & 3 & 2 & 2 & 3 & 2 & & 2 & 1 & 2 \\
N11 & 2 & 2 & 2 & 3 & 1 & 3 & 3 & 1 & 1 & 2 & & 2 \\
N12 & 3 & 3 & 3 & 3 & 3 & 3 & 1 & 2 & 2 & 1 & 2 & 2 \\
N13 & 3 & 3 & 3 & 1 & 3 & 2 & 2 & 2 & 2 & 2 & 2 & 2 \\
N14 & 3 & 3 & 3 & 2 & 2 & 2 & 2 & 2 & 2 & 1 & 2 & 2 \\
\hline
\end{tabular}

the solution that is based on the parameters of Table 8 is worse than the proposed PSO.

The best schedule (construction of the building of nuclear power plant) is as follows:

$$
\begin{aligned}
& \text { Construction team } 1(\mathrm{~N} 7==>\mathrm{N} 10==>\mathrm{N} 12) \\
& \text { Construction team } 2(\mathrm{~N} 8==>\mathrm{N} 2) . \\
& \text { Construction team } 3(\mathrm{~N} 9==>\mathrm{N} 3==>\mathrm{N} 14) . \\
& \text { Construction team } 4(\mathrm{~N} 1==>\mathrm{N} 5==>\mathrm{N} 4) . \\
& \text { Construction team } 5(\mathrm{~N} 11==>\mathrm{N} 13==>\mathrm{N} 6) .
\end{aligned}
$$

The best schedule (reactor installation) is as follows:

$$
\begin{aligned}
& \text { Reactor installation team } 1(\mathrm{~N} 8==>\mathrm{N} 13==>\mathrm{N} 10) \\
& \text { Reactor installation team } 2(\mathrm{~N} 4==>\mathrm{N} 14==>\mathrm{N} 12) \text {. } \\
& \text { Reactor installation team } 3(\mathrm{~N} 6==>\mathrm{N} 11) . \\
& \text { Reactor installation team } 4(\mathrm{~N} 3==>\mathrm{N} 2==>\mathrm{N} 9) \text {. } \\
& \text { Reactor installation team } 6(\mathrm{~N} 5==>\mathrm{N} 1==>\mathrm{N} 7) \text {. } \\
& \text { The minimum delay: } 136.7
\end{aligned}
$$

\section{Conclusion}

This paper proposed a particle swarm optimization algorithm that integrates building construction scheduling and reactor installation scheduling to effectively solve the optimization problem of nuclear power plant construction scheduling. The objective function was to minimize the delay. We can see that the increase of construction teams is more effective than the increase of reactor installation team. When the range between lower bound and upper bound becomes wider, the performance becomes better. A mathematical model of the integrated problem was generated using LINGO 11.0 to obtain the optimum solution. The proposed method, applied in LINGO 11.0, obtained the optimal solution. Simulations have been conducted to show the superior performance of our method in comparison with a greedy scheduling method under various network scenarios.

\section{Conflict of Interests}

The authors declare that there is no conflict of interests regarding the publication of this paper.

\section{Acknowledgment}

Kuo-Yu Tsai is supported by Ministry of Science and Technology, Taiwan, under Grant nos. 103-2221-E-146-005-MY2, 103-2221-E-011-090-MY2, and 104-2119-M-011-003-.

\section{References}

[1] Plans For New Reactors Worldwide, http://www.world-nuclear .org/info/Current-and-Future-Generation/Plans-For-New-Reactors-Worldwide/.

[2] V. E. Whisker, A. J. Baratta, S. Yerrapathruni et al., "Using immersive virtual environments to develop and visualize construction schedules for advanced nuclear power plants," in Proceedings of the 2nd International Congress on Advances in Nuclear Power Plants (ICAPP '03), Cordoba, Spain, May 2003.

[3] Y. Jung and G. E. Gibson Jr., "Planning for computer integrated construction," Journal of Computing in Civil Engineering, vol. 13, no. 4, pp. 217-225, 1999.

[4] N. Dawood and E. Sriprasert, "Construction scheduling using multi-constraint and genetic algorithms approach," Construction Management and Economics, vol. 24, no. 1, pp. 19-30, 2006.

[5] S. Kaiafa and A. P. Chassiakos, "A genetic algorithm for resource allocation in construction projects," in Proceedings of the Creative Construction Conference, pp. 386-391, Kraków, Poland, June 2015.

[6] S.-S. Leu and C.-H. Yang, "A genetic-algorithm-based resourceconstrained construction scheduling system," Construction Management and Economics, vol. 17, no. 6, pp. 767-776, 1999.

[7] R. B. Harris and P. G. Ioannou, "Scheduling projects with repeating activities," Journal of Construction Engineering and Management, vol. 124, no. 4, pp. 269-278, 1998.

[8] J.-L. Kim, "Integrated genetic algorithm and its applications for construction resource optimization," in Proceedings of the 43rd Winter Simulation Conference (WSC '10), pp. 3212-3219, IEEE, Baltimore, Md, USA, December 2010. 
[9] W. Y. Thabet and Y. J. Beliveau, "HVLS: horizontal and vertical logic scheduling for multistory projects," Journal of Construction Engineering and Management, vol. 120, no. 4, pp. 875-892, 1994.

[10] J. Zhou, P. E. D. Love, X. Wang, K. L. Teo, and Z. Irani, “A review of methods and algorithms for optimizing construction scheduling," Journal of the Operational Research Society, vol. 64, no. 8, pp. 1091-1105, 2013.

[11] A. B. Senouci and H. R. Al-Derham, "Genetic algorithm-based multi-objective model for scheduling of linear construction projects," Advances in Engineering Software, vol. 39, no. 12, pp. 1023-1028, 2008.

[12] L. D. Long and A. Ohsato, "A genetic algorithm-based method for scheduling repetitive construction projects," Automation in Construction, vol. 18, no. 4, pp. 499-511, 2009.

[13] V. Faghihi, K. F. Reinschmidt, and J. H. Kang, "Construction scheduling using genetic algorithm based on building information model," Expert Systems with Applications, vol. 41, no. 16, pp. 7565-7578, 2014.

[14] T.-K. Lim, C.-Y. Yi, D.-E. Lee, and D. Arditi, "Concurrent construction scheduling simulation algorithm," Computer-Aided Civil and Infrastructure Engineering, vol. 29, no. 6, pp. 449-463, 2014.

[15] R. Sonmez and F. Uysal, "Backward-forward hybrid genetic algorithm for resource-constrained multiproject scheduling problem," Journal of Computing in Civil Engineering, vol. 29, no. 5, pp. 1509-1512, 2015.

[16] S.-S. Leu and C.-H. Yang, "GA-based multicriteria optimal model for construction scheduling," Journal of Construction Engineering and Management, vol. 125, no. 6, pp. 420-427, 1999.

[17] A. B. Senouci and N. N. Eldin, "Use of genetic algorithms in resource scheduling of construction projects," Journal of Construction Engineering and Management, vol. 130, no. 6, pp. 869-877, 2004.

[18] A. Agarwal, S. Colak, and S. Erenguc, "A Neurogenetic approach for the resource-constrained project scheduling problem," Computers \& Operations Research, vol. 38, no. 1, pp. 44-50, 2011.

[19] M. Amiri, M. Zandieh, M. Yazdani, and A. Bagheri, "A variable neighbourhood search algorithm for the flexible jobshop scheduling problem," International Journal of Production Research, vol. 48, no. 19, pp. 5671-5689, 2010.

[20] P. Hansen and N. Mladenovic, "Variable neighborhood search: principles and applications," European Journal of Operational Research, vol. 130, no. 3, pp. 449-467, 2001.

[21] N. Mladenović and P. Hansen, "Variable neighborhood search," Computers \& Operations Research, vol. 24, no. 11, pp. 1097-1100, 1997. 

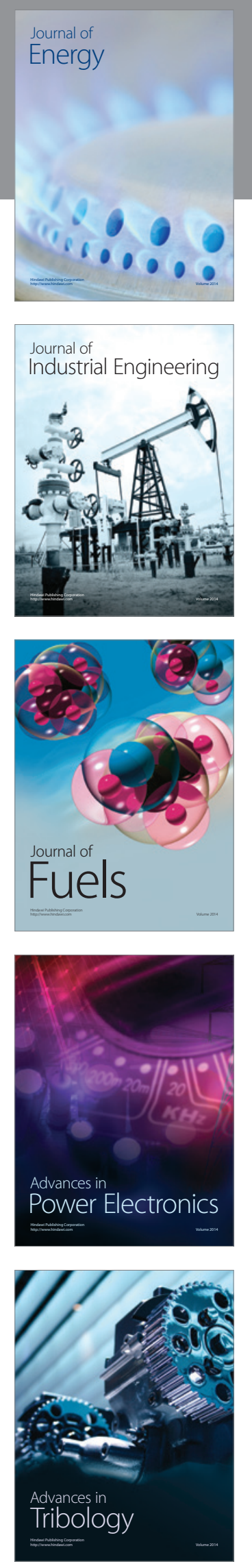
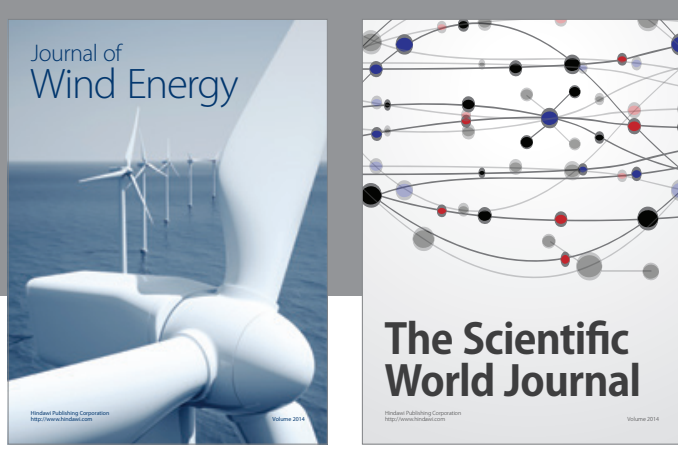

The Scientific World Journal
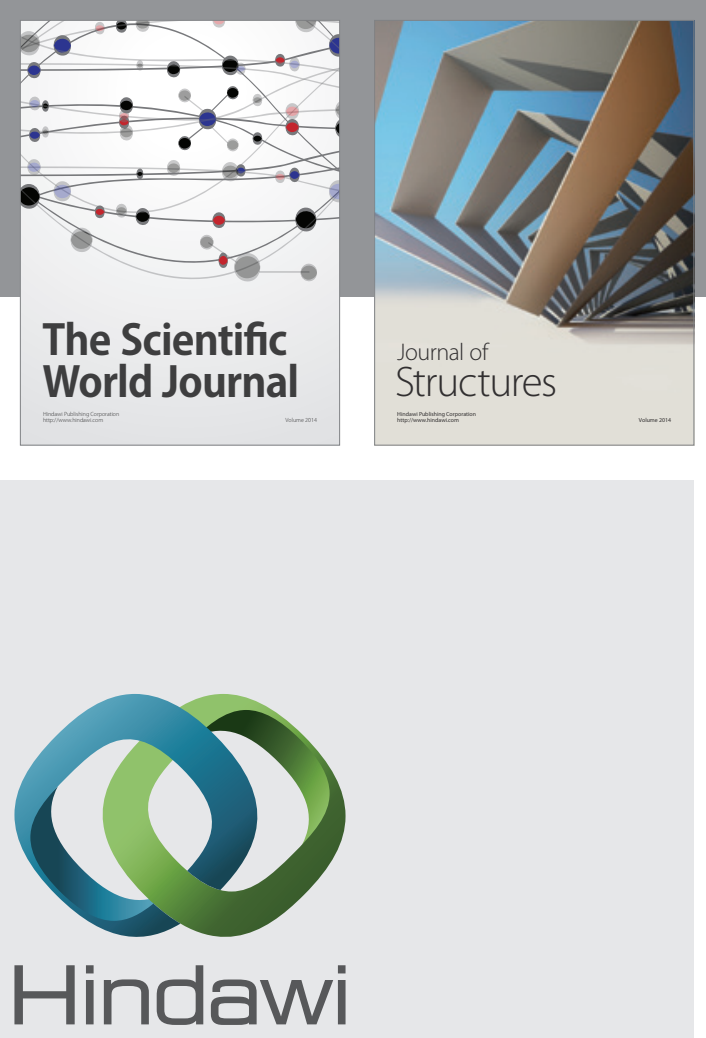

Submit your manuscripts at

http://www.hindawi.com
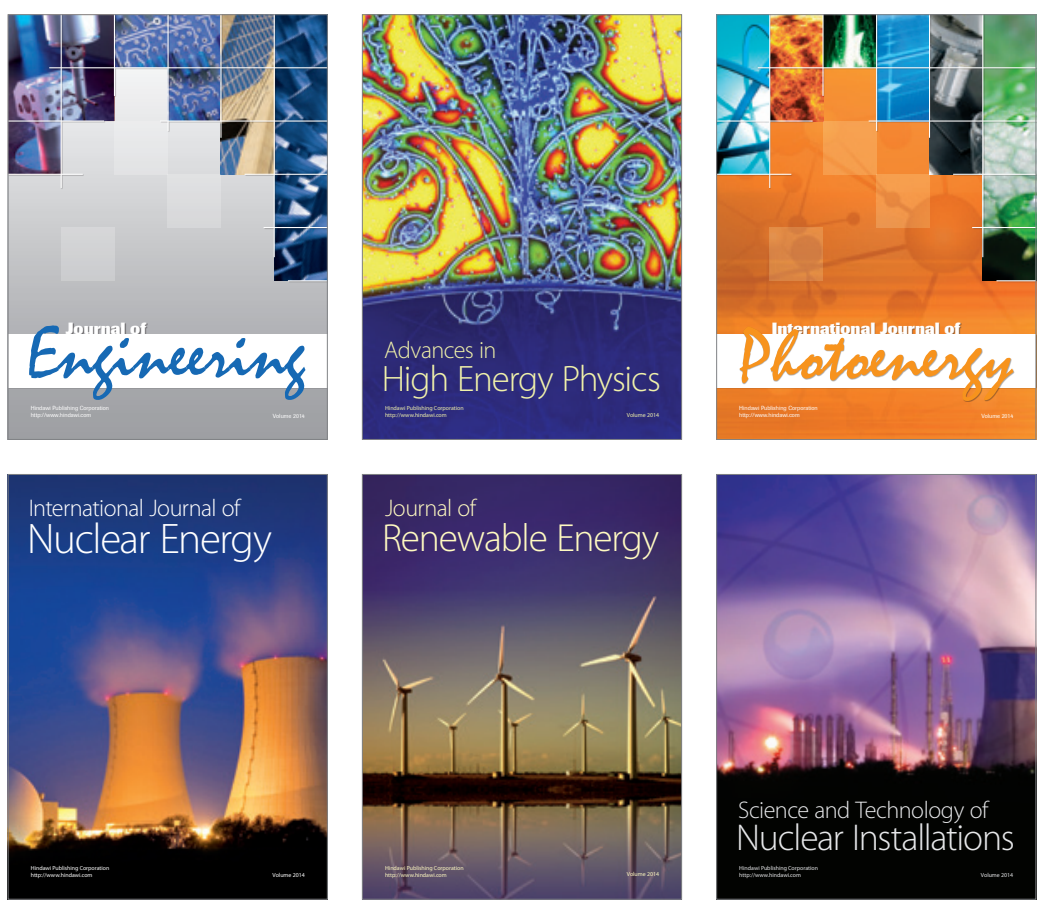
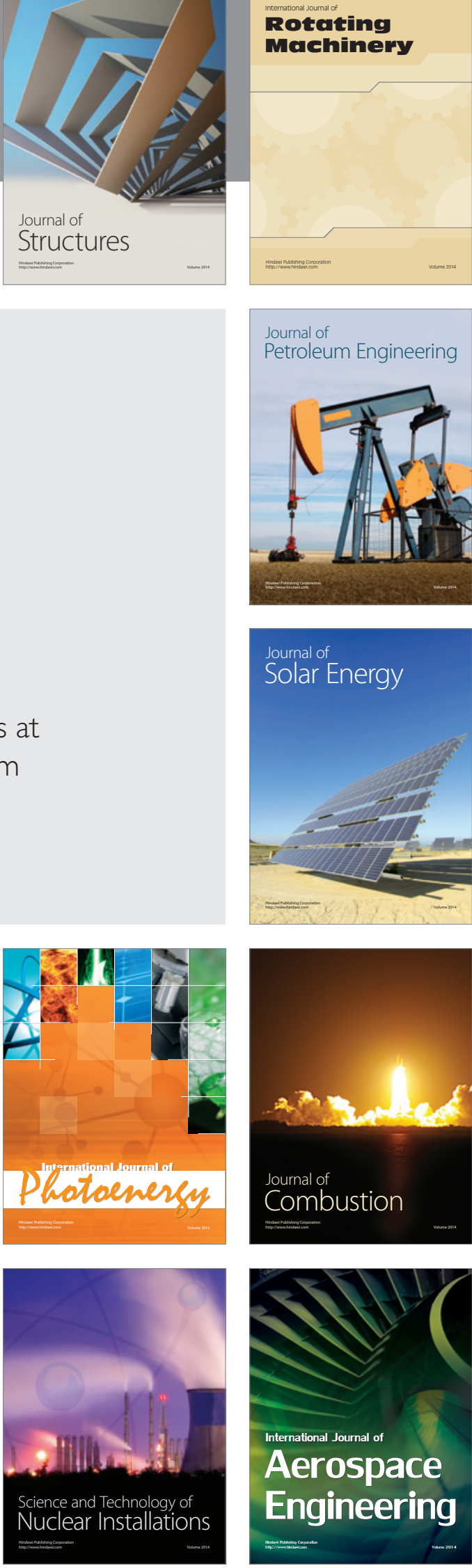